\title{
A Stochastic Formulation of the Bass Model of New-Product Diffusion
}

\author{
SHUN-CHEN NIU* \\ School of Management, The University of Texas at Dallas, P.O. Box 830688, Richardson, \\ TX 75083-0688, USA
}

(Received 7 August 2002; In final form 29 August 2002)

\begin{abstract}
For a large variety of new products, the Bass Model (BM) describes the empirical cumulative-adoptions curve extremely well. The BM postulates that the trajectory of cumulative adoptions of a new product follows a deterministic function whose instantaneous growth rate depends on two parameters, one of which captures an individual's intrinsic tendency to purchase, independent of the number of previous adopters, and the other captures a positive force of influence on an individual by previous adopters. In this paper, we formulate a stochastic version of the BM, which we call the Stochastic Bass Model (SBM), where the trajectory of cumulative number of adoptions is governed by a pure birth process. We show that with an appropriately-chosen set of birth rates, the fractions of individuals who have adopted the product by time $t$ in a family of SBMs indexed by the size of the target population converge in probability to the deterministic fraction in a corresponding BM, when the population size approaches infinity. The formulation therefore supports and expands the BM by allowing stochastic trajectories.
\end{abstract}

Key words: Pure birth processes; Diffusion models; New-product adoptions; Epidemics

AMS 2000 Subject Classification: Primary: 92D25; Secondary: 92D30, 60J80

\section{INTRODUCTION}

In the past several decades, new-product diffusion models has been an active area of research in marketing (Mahajan et al. [11]; Mahajan and Wind [12]; and Rogers [16]). Such models are useful because they can provide insights into the timing of initial purchase of new products by consumers. Much of the work in this area has been spawned by an influential paper of Bass [5], in which it was assumed that the instantaneous rate of adoption of a new product (or technology) at any time epoch depends on two forces, one is an intrinsic tendency for an individual to make a purchase (given that the individual has not yet adopted), independent of the number of previous adopters in the target population, and the other is a positive influence by previous adopters on the remaining individuals in the population (via, e.g., word of mouth).

The mathematical formulation of the Bass Model (BM) is as follows. Let $p$ and $q$ be two parameters that represent the extent of the above-mentioned two forces, let $m$ be the size of a target population, and let $N(t)$ be the cumulative number of adopters of a new product by time $t$. Then, under the assumption that $N(t)$ is a continuous function

*E-mail: scniu@utdallas.edu 
with $N(0)=0$, Bass postulates (Bass [5], p. 217) that the following differential equation holds:

$$
\frac{\mathrm{d} N(t)}{\mathrm{d} t}=[m-N(t)]\left[p+\frac{q}{m} N(t)\right], \quad t \geq 0 .
$$

That is, the growth rate of $N(t)$ at time $t$ is equal to the product of $m-N(t)$ and $p+(q / m) N(t)$, where $m-N(t)$ is the size of the remaining population and $p+(q / m) N(t)$ is the instantaneous adoption rate of every individual in the remaining population.

Notice that if we let $F(t)$ be the (continuous) fraction of individuals who have adopted the product by time $t$, i.e., let

$$
F(t) \equiv \frac{N(t)}{m}
$$

then, Eq. (1) has the following equivalent form:

$$
f(t)=[1-F(t)][p+q F(t)], \quad t \geq 0,
$$

where $f(t)$ denotes the derivative of $F(t)$. It was shown in Bass [5] that the solution of (3) is given by

$$
F(t)=\frac{1-e^{-(p+q) t}}{1+(q / p) e^{-(p+q) t}}, \quad t \geq 0,
$$

and that this S-shaped solution (or equivalently, its bell-shaped derivative $f(t)$ ) provides excellent empirical fit for the timing of initial purchase for a wide range of consumer durables.

While the deterministic BM is parsimonious, there also has been a common belief (see, e.g., Eliashberg and Chatterjee [7]) that it would be of interest to have an appropriate stochastic version of his model. In this paper, we formulate a stochastic counterpart of the $\mathrm{BM}$ where the trajectory of cumulative number of adoptions is governed by a pure birth process. Our purpose is to show that with an appropriately-chosen set of birth rates, the fractions of individuals who have adopted the product by time $t$ in a family of the formulated stochastic Bass models indexed by the population size $m$ converge in probability to the solution (4) of a corresponding BM, when $m$ approaches infinity.

Our formulation is inspired by a model introduced by Taga and Isii [19] for the study of the pattern of communication between an information source and individuals within a social group. Specifically, Taga and Tsii assume that transmission of a given piece of news can take place either directly from the source to an individual or between individuals within the group; and that the growth of the number of individuals who have received the news follows a pure birth process whose birth rates are functions of two parameters that correspond to these two modes of information transmission. Observe that while the intended application context is different, the stochastic assumptions in Taga and Isii's model are remarkably similar in spirit to the deterministic ones in the BM. A primary motivation of this paper is to show that with a proper scaling of the birth rates in Taga and Isii's model, this observation can be formalized as a convergence result. By bridging these two families of models together, we hope to gain a better understanding of the $\mathrm{BM}$ in terms of having micro-level stochastic interactions amongst individual adopters (see, e.g., Roberts and Lattin [15]). 
In Section 2, we describe the model and state the convergence result; the proof is given in Section 3. In Section 4, we comment on future work.

\section{THE MODEL AND THE CONVERGENCE RESULT}

Consider a product that has a potential market size of $m$ individuals. We assume that each individual in this potential market, which will be referred to as the target population, will eventually adopt (or purchase) exactly one unit of the product. The timing of this adoption is, however, uncertain. Let $A_{m}(t)$ be the cumulative number of adoptions by time $t$, with $A_{m}(0) \equiv 0$. Following Taga and Isii [19], we assume $\left\{A_{m}(t), t \geq 0\right\}$ is a pure birth process. Our specific assumptions on the birth rates are described as follows.

If an individual has not yet adopted the product by time $t$, then we assume that the "intrinsic" probability for this individual to adopt the product during the time interval $(t, t+h)$ is (independently of everything else) given by

$$
\alpha h+o(h)
$$

If, on the other hand, an individual has already adopted the product by time $t$, then we assume that the probability for this individual to "induce" any other member of the remaining population at time $t$ to adopt in $(t, t+h)$ is (independently of everything else) given by

$$
\frac{\beta}{m-1} h+o(h)
$$

(If $m=1$, we define $\beta /(m-1)$ as 0 .)

Thus, each individual in the target population has an intrinsic adoption rate and an induction rate, given by $\alpha$ and $\beta$ respectively; moreover, the induction rate $\beta$ associated with each individual is apportioned uniformly to all other members of the population. The parameters $\alpha$ and $\beta$ correspond conceptually to the parameters $p$ and $q$ in the deterministic BM.

Suppose $A_{m}(t)=j$, where $0 \leq j \leq m-1$. Then, according to (5) and (6), the probability for any individual in the remaining population at time $t$ to adopt the product in $(t, t+h)$ is given by $[\alpha+j \beta /(m-1)] h+o(h)$. Since the size of this remaining population equals $m-j$, the probability for $A_{m}(t)$ to increase to $j+1$ (from $j$ ) in $(t, t+h)$ is given by $\lambda_{m j} h+o(h)$, where

$$
\lambda_{m j} \equiv(m-j)\left(\alpha+\frac{\beta}{m-1} j\right), \quad j=0,1, \ldots, m-1 .
$$

Since the growth of $A_{m}(t)$ stops upon reaching level $m$, it follows that the range for $j$ in (7) can be extended to cover the case $j=m$ as well. We will refer to $\lambda_{m j}$ as the birth (or diffusion) rate at state $j$, and the resulting pure birth process $\left\{A_{m}(t), t \geq 0\right\}$ with birth rates $\left\{\lambda_{m j}\right\}_{j=0}^{m}$ as the Stochastic Bass Model (SBM).

Paralleling (2), define

$$
B_{m}(t) \equiv \frac{A_{m}(t)}{m} ;
$$


that is, let $B_{m}(t)$ be the fraction of individuals who have adopted the product by time $t$ in an SBM with population size $m$. Then, our result is the following weak law for $B_{m}(t)$.

THEOREM For $t \geq 0$ and any positive $\varepsilon$,

$$
\lim _{m \rightarrow \infty} P\left\{\left|B_{m}(t)-F_{\infty}(t)\right|>\varepsilon\right\}=0
$$

where $F_{\infty}(t)$ is given by

$$
F_{\infty}(t)=\frac{1-e^{-(\alpha+\beta) t}}{1+(\beta / \alpha) e^{-(\alpha+\beta) t}},
$$

the solution of (3) in a corresponding BM with parameters $p=\alpha$ and $q=\beta$.

We note that the difference between the SBM and Taga and Isii's original formulation is that in the latter, the probability in (6) is defined as $\beta h+o(h)$ (Taga and Isii [19], pp. 27-28). The apportionment, or scaling, of $\beta$ in the SBM parallels the term $q / m$ in (1) (apart from using $m-1$ in place of $m$ ); and it is this scaling of Taga and Isii's model that accounts for the convergence in (9).

The SBM is also closely tied to several well-known models in the theory of epidemics (Bailey [2]; Bartholomew [3], Chapters 9 and 10; Bartlett [4]; and Gani [9]). In particular, with $\alpha=\beta$ and one initial adopter, but without the factor $m-1$ in (6), the SBM becomes the simple stochastic epidemic or the logistic model (Bailey [1]; Haskey [10]; and Mansfield and Hensley [13]). The simple stochastic epidemic model with a large population of susceptibles has been studied in Williams [20]. Williams observed that the epidemic curve (i.e., the derivative of expected cumulative number of infectives over time) is quite different from that in a corresponding deterministic model (see also Feller [8]), but succeeded in showing that if the number of initial infectives is also allowed to increase, then there is asymptotic agreement.

\section{PROOF}

The proof consists of two steps. In the first, we show that

$$
\lim _{m \rightarrow \infty} E\left[B_{m}(t)\right]=F_{\infty}(t)
$$

and in the second, we show that

$$
\lim _{m \rightarrow \infty} \operatorname{Var}\left[B_{m}(t)\right]=0 .
$$

The convergence in (9) is then a standard consequence of Markov's inequality:

$$
\begin{aligned}
P\left\{\left|B_{m}(t)-F_{\infty}(t)\right|>\varepsilon\right\} & \leq \frac{E\left\{\left[B_{m}(t)-F_{\infty}(t)\right]^{2}\right\}}{\varepsilon^{2}} \\
& =\frac{\operatorname{Var}\left[B_{m}(t)\right]+\left\{E\left[B_{m}(t)\right]-F_{\infty}(t)\right\}^{2}}{\varepsilon^{2}}
\end{aligned}
$$

Below, we will establish (11) and (12) via several intermediate lemmas. 
We begin with the observation that $E\left[B_{m}(t)\right]=F_{m}(t)$, where $F_{m}(t)$ denotes the distribution of the adoption time of an "arbitrary" individual in the target population. This follows because if $A_{m}(t)=k$, where $0 \leq k \leq m$, then the conditional probability for a randomlyselected individual to have adopted the product by time $t$ equals $k / m$. We will henceforth replace $E\left[B_{m}(t)\right]$ by the equivalent notation $F_{m}(t)$ and work with the associated adoption-time interpretation.

Next, we note that explicit formulas for $F_{m}(t)$, for any $m \geq 1$, can be obtained from standard results for the state distribution of pure birth processes (Bartlett [4], Section 3.2; Taga and Isii [19], p. 28; Bartholomew [3], p. 252; or Ross [17], p. 324). Specifically, it can be shown that $F_{m}(t)$ has the form:

$$
F_{m}(t)=1-\sum_{i=0}^{m-1} a_{m i} e^{-\lambda_{m i} t}, \quad t \geq 0,
$$

where the $a_{m i}$ s are functions of the birth rates. As examples, we have (details omitted):

$$
\begin{aligned}
& F_{1}(t)=1-e^{-\alpha t}, \\
& F_{2}(t)=1-\frac{-\beta}{\alpha-\beta} e^{-2 \alpha t}-\frac{\alpha}{\alpha-\beta} e^{-(\alpha+\beta) t}, \\
& F_{3}(t)=1-\frac{\beta^{2}}{(2 \alpha-\beta)(\alpha-\beta)} e^{-3 \alpha t}-\frac{-\beta}{\alpha-\beta} e^{-2(\alpha+\beta / 2) t}-\frac{2 \alpha+\beta}{2 \alpha-\beta} e^{-(\alpha+\beta) t},
\end{aligned}
$$

and

$$
\begin{aligned}
F_{4}(t)= & 1-\frac{-2 \beta^{3}}{(\alpha-\beta)(3 \alpha-\beta)(3 \alpha-2 \beta)} e^{-4 \alpha t}-\frac{2 \beta^{2}}{(\alpha-\beta)(3 \alpha-\beta)} e^{-3(\alpha+\beta / 3) t} \\
& -\frac{-9 \beta}{(3 \alpha-2 \beta)(3 \alpha-\beta)} e^{-2(\alpha+2 \beta / 3) t}-\frac{3 \alpha+2 \beta}{3 \alpha-\beta} e^{-(\alpha+\beta) t}
\end{aligned}
$$

The expressions, however, become extremely complicated as $m$ increases. The key idea in our proof of (11) is to bound the $F_{m}$ s from above by the solutions of a corresponding family of BMs.

Denote by $f_{m}(t)$ the density of $F_{m}(t)$. The starting point of our bounding argument is the following lemma.

LEMMA 1 For $m=1$, we have

$$
f_{1}(t)=\alpha\left[1-F_{1}(t)\right], \quad t \geq 0
$$

and for $m \geq 2$, we have

$$
f_{m}(t) \leq\left[1-F_{m}(t)\right]\left[\alpha+\beta_{m} F_{m}(t)\right], \quad t \geq 0
$$

where

$$
\beta_{m} \equiv \frac{m \beta}{m-1} .
$$


Proof The statement for $m=1$ is an immediate consequence of the fact that $F_{1}$ is the exponential distribution with parameter $\alpha$.

We now assume $m \geq 2$. With $A_{m}(t)$ replacing $j$ in (7), we have

$$
\lambda_{m A_{m}(t)}=\left[m-A_{m}(t)\right]\left[\alpha+\frac{\beta}{m-1} A_{m}(t)\right]
$$

and upon taking expectations and dividing by $m$, this becomes

$$
\frac{E\left[\lambda_{\left.m A_{m}(t)\right]}\right.}{m}=\alpha \frac{E\left[m-A_{m}(t)\right]}{m}+\beta \frac{E\left\{\left[m-A_{m}(t)\right] A_{m}(t)\right\}}{m(m-1)} .
$$

By conditioning on $A_{m}(t)$, we have

$$
\frac{E\left[\lambda_{m A_{m}(t)}\right]}{m}=\frac{1}{m} \sum_{k=0}^{m-1} \lambda_{m k} P\left\{A_{m}(t)=k\right\} .
$$

For $1 \leq j \leq m$, denote by $f_{m j}(t)$ the density function of the $j$ th adoption epoch; then, we clearly have:

$$
\lambda_{m k} P\left\{A_{m}(t)=k\right\}=f_{m, k+1}(t)
$$

and

$$
\frac{1}{m} \sum_{j=1}^{m} f_{m j}(t)=f_{m}(t)
$$

Therefore,

$$
\frac{E\left[\lambda_{m A_{m}(t)}\right]}{m}=f_{m}(t)
$$

Next, note that

$$
\frac{E\left[m-A_{m}(t)\right]}{m}=1-\frac{E\left[A_{m}(t)\right]}{m}=1-F_{m}(t) .
$$

Finally, since $E\left\{\left[A_{m}(t)\right]^{2}\right\} \geq\left\{E\left[A_{m}(t)\right]\right\}^{2}$, we have

$$
E\left\{\left[m-A_{m}(t)\right] A_{m}(t)\right\} \leq E\left[m-A_{m}(t)\right] E\left[A_{m}(t)\right] ;
$$

and therefore,

$$
\begin{aligned}
\frac{E\left\{\left[m-A_{m}(t)\right] A_{m}(t)\right\}}{m(m-1)} & \leq \frac{m}{m-1} \frac{E\left[m-A_{m}(t)\right]}{m} \frac{E\left[A_{m}(t)\right]}{m} \\
& =\frac{m}{m-1}\left[1-F_{m}(t)\right] F_{m}(t) .
\end{aligned}
$$

Substitution of (17), (18), and (19) into (16) now establishes (13). 
Observe that if we replace the inequality in (13) with an equality, then we will have a corresponding differential equation that is of the same form as (3). This observation naturally suggests that we compare $F_{m}$ against the solution of this corresponding differential equation.

Formally, define, for every $m \geq 2$, a BM with parameters $p=\alpha$ and $q=\beta_{m}$; and denote by $G_{m}(t)$ the fraction of individuals who have adopted by time $t$ in this model. Then, according to (3), the $G_{m}$ s satisfy

$$
g_{m}(t)=\left[1-G_{m}(t)\right]\left[\alpha+\beta_{m} G_{m}(t)\right], \quad t \geq 0,
$$

where $g_{m}(t)$ denotes the derivative of $G_{m}(t)$; moreover, in light of (4), we have

$$
G_{m}(t)=\frac{1-e^{-\left(\alpha+\beta_{m}\right) t}}{1+\left(\beta_{m} / \alpha\right) e^{-\left(\alpha+\beta_{m}\right) t}}
$$

To have full correspondence between the $G_{m} \mathrm{~s}$ and the $F_{m} \mathrm{~s}$, we further define

$$
G_{1}(t) \equiv \begin{cases}1, & \text { for } t \geq 0 \\ 0, & \text { otherwise }\end{cases}
$$

The function $G_{1}$ can be viewed as the limiting solution of the BM when $q \rightarrow \infty$ (for any fixed $p$ ).

Let $D_{1}$ and $D_{2}$ be two distribution functions and denote by $\bar{D}_{1}$ and $\bar{D}_{2}$, respectively, their corresponding tail distributions (i.e., let $\bar{D}_{i}(t) \equiv 1-D_{i}(t)$ for $\left.i=1,2\right)$. Recall that $D_{1}$ is said to be stochastically less than $D_{2}$ whenever the inequality $\bar{D}_{1}(t) \leq \bar{D}_{2}(t)$ holds for all $t \geq 0$. In the next lemma, we show that $G_{m}$ is stochastically less than $F_{m}$.

LEMMA 2 For all $m \geq 1$, we have

$$
\bar{G}_{m}(t) \leq \bar{F}_{m}(t), \quad t \geq 0 .
$$

Proof Since $\bar{G}_{1}(t)=0$ for all $t \geq 0,(21)$ is clearly true for $m=1$.

For $m \geq 2$, we first rewrite (13) as

$$
\frac{f_{m}(t)}{\left[1-F_{m}(t)\right]\left[\alpha+\beta_{m} F_{m}(t)\right]} \leq 1 .
$$

Next, it is easily seen that the left-hand side of (22) can be expanded as:

$$
\frac{1}{\alpha+\beta_{m}} \frac{f_{m}(t)}{1-F_{m}(t)}+\frac{\beta_{m}}{\alpha+\beta_{m}} \frac{f_{m}(t)}{\alpha+\beta_{m} F_{m}(t)} .
$$

Now, substituting (23) into (22) and integrating both sides of the resulting inequality from 0 to $t$ yields (after a little bit of algebra)

$$
-\frac{1}{\alpha+\beta_{m}} \ln \left(\frac{1-F_{m}(t)}{1+\left(\beta_{m} / \alpha\right) F_{m}(t)}\right) \leq t .
$$


It follows that

$$
\frac{1-F_{m}(t)}{1+\left(\beta_{m} / \alpha\right) F_{m}(t)} \geq e^{-\left(\alpha+\beta_{m}\right) t}
$$

which, after a rearrangement, becomes

$$
F_{m}(t) \leq \frac{1-e^{-\left(\alpha+\beta_{m}\right) t}}{1+\left(\beta_{m} / \alpha\right) e^{-\left(\alpha+\beta_{m}\right) t}}
$$

Since the right-hand side of $(24)$ is $G_{m}(t)$ (see (20)), this proves (21).

Lemma 2 can also be rephrased as that the function $G_{m}$ lies entirely above the function $F_{m}$ for every $m$. In the next lemma, we consider the region bounded between $G_{m}$ and $F_{m}$; and we show that as a function of $m$, the areas of these regions converge to 0 when $m$ increases to infinity.

LEMMA 3

$$
\lim _{m \rightarrow \infty} \int_{0}^{\infty}\left[G_{m}(t)-F_{m}(t)\right] \mathrm{d} t=0
$$

Moreover, the convergence is monotone.

Proof Clearly,

$$
\int_{0}^{\infty}\left[G_{m}(t)-F_{m}(t)\right] \mathrm{d} t=\int_{0}^{\infty}\left[\bar{F}_{m}(t)-\bar{G}_{m}(t)\right] \mathrm{d} t .
$$

Denote by $\mu_{D}$ the mean of a given distribution function $D$, and recall the standard formula $\mu_{D}=\int_{0}^{\infty} \bar{D}(t) \mathrm{d} t$. Then, the right-hand side of (25) can be evaluated as $\mu_{F_{m}}-\mu_{G_{m}}$, provided that both $\mu_{F_{m}}$ and $\mu_{G_{m}}$ are finite. We will examine $\mu_{F_{m}}$ and $\mu_{G_{m}}$ separately.

We begin with $\mu_{G_{m}}$. Since $\mu_{G_{1}}=0$, we will consider $\mu_{G_{m}}$ for $m \geq 2$. From (4), it easily follows that

$$
\bar{F}(t)=\frac{(p+q) e^{-(p+q) t}}{p+q e^{-(p+q) t}} .
$$

By differentiating (26) with respect to $q$, it is straightforward to show that $\bar{F}(t)$ is strictly decreasing in $q$ (for fixed $p$ and $t$ ). Since $\beta_{m}$ (see (14)) is strictly decreasing in $m$ with $\lim _{m \rightarrow \infty} \beta_{m}=\beta$ and since $\bar{F}(t)$ is continuous in $\underline{q}$, it follows from (20) that for all $t \geq 0$, the $\bar{G}_{m}(t)$ s converge monotonically from below to $\bar{F}_{\infty}(t)$, where $F_{\infty}(t)$ is given by $(10)$. With $p=\alpha$ and $q=\beta$ in (26), it is easily shown that

$$
\mu_{F_{\infty}}=\int_{0}^{\infty} \bar{F}_{\infty}(t) \mathrm{d} t=\frac{1}{\beta} \ln \left(\frac{\alpha+\beta}{\alpha}\right)
$$


and with $\beta_{m}$ replacing $\beta$ in (27), we also have

$$
\mu_{G_{m}}=\int_{0}^{\infty} \bar{G}_{m}(t) \mathrm{d} t=\frac{1}{\beta_{m}} \ln \left(\frac{\alpha+\beta_{m}}{\alpha}\right) .
$$

It follows that $\mu_{G_{m}}$ is strictly increasing in $m$ with

$$
\lim _{m \rightarrow \infty} \mu_{G_{m}}=\mu_{F_{\infty}}<\infty
$$

We now turn our attention to $\mu_{F_{m}}$. For $1 \leq j \leq m$, denote by $A_{m j}$ the $j$ th adoption epoch; then,

$$
\mu_{F_{m}}=\frac{1}{m} \sum_{j=1}^{m} E\left[A_{m j}\right] .
$$

Next, for $1 \leq i \leq m$, denote by $X_{m i}$ the $i$ th inter-adoption time; then, since the $X_{m i}$ s are exponentially distributed and since $A_{m j}=\sum_{i=1}^{j} X_{m i}$, we also have

$$
E\left[A_{m j}\right]=\sum_{i=0}^{j-1} \frac{1}{\lambda_{m i}},
$$

where the $\lambda_{m i}$ s are given by (7). Substitution of (30) into (29) now yields, after an interchange of the order of summation,

$$
\mu_{F_{m}}=\frac{1}{m} \sum_{i=0}^{m-1} \frac{1}{\alpha+[\beta /(m-1)] i} .
$$

It follows easily from (31) that $\mu_{F_{1}}=1 / \alpha$ and

$$
\mu_{F_{2}}=\frac{1}{2}\left(\frac{1}{\alpha}+\frac{1}{\alpha+\beta}\right)
$$

therefore, we have $\mu_{F_{1}}>\mu_{F_{2}}$. We will next consider $\mu_{F_{m}}$ for $m \geq 2$, and prove that the sequence of $\mu_{F_{m}} \mathrm{~s}$ decreases monotonically to $\mu_{F_{\infty}}$. (Actually, we conjecture that the $F_{m} \mathrm{~s}$ decrease stochastically to $F_{\infty}$, but have been unable to prove this stronger result.)

For $0 \leq y \leq 1$, define

$$
\phi(y) \equiv \frac{1}{\alpha+\beta y}
$$

and observe that in terms of the function $\phi$, (31) can be written as $\mu_{F_{m}}=E\left[\phi\left(U_{m}\right)\right]$, where $U_{m}$ is distributed uniformly over $i /(m-1)$ for $i=0,1, \ldots, m-1$. Now, consider $U_{m}$ and $U_{m+1}$, for any $m \geq 2$; and observe further that the probability-mass function of $U_{m}$ is, intuitively, more "spread out" than that of $U_{m+1}$. Since the function $\phi$ is strictly convex in $y$, these observations naturally suggest that the following inequality should hold:

$$
E\left[\phi\left(U_{m}\right)\right]>E\left[\phi\left(U_{m+1}\right)\right] .
$$

We will prove (32) via a coupling argument. 
The first step is to construct $U_{m}$ from $U_{m+1}$ as follows. Let $U_{m}=U_{m+1}$ if $U_{m+1}$ equals either 0 or 1 ; and let

$$
U_{m}= \begin{cases}\frac{i-1}{m-1}, & \text { with probability } \frac{i}{m} \\ \frac{i}{m-1}, & \text { with probability } 1-\frac{i}{m}\end{cases}
$$

if $U_{m+1}=i / m$, for $i=1, \ldots, m-1$. Note that for this construction to be valid, the resulting $U_{m}$ must satisfy $P\left\{U_{m}=i /(m-1)\right\}=1 / m$ for all $i=0,1, \ldots, m-1$; this can be easily verified, and we omit the details.

Next, observe that the coupling above can be restated as

$$
U_{m}=U_{m+1}+Z_{m+1}
$$

where, by definition, $Z_{m+1}=0$ if $U_{m+1}$ equals either 0 or 1 , and

$$
Z_{m+1}= \begin{cases}-\frac{m-i}{m(m-1)}, & \text { with probability } \frac{i}{m} \\ \frac{i}{m(m-1)}, & \text { with probability } 1-\frac{i}{m}\end{cases}
$$

if $U_{m+1}=i / m$, for $i=1, \ldots, m-1$. Moreover, it is easily shown that we have

$$
E\left[Z_{m+1} \mid U_{m+1}\right]=0
$$

with probability 1 . (Relations (33) and (34) show that $U_{m}$ is greater than $U_{m+1}$ in the sense of what is known as convex order.) It now follows in a standard manner from (33), Jensen's inequality, and (34) that

$$
\begin{aligned}
E\left[\phi\left(U_{m}\right)\right] & =E\left[\phi\left(U_{m+1}+Z_{m+1}\right)\right] \\
& =E\left[E\left[\phi\left(U_{m+1}+Z_{m+1}\right) \mid U_{m+1}\right]\right] \\
& >E\left[\phi\left(U_{m+1}+E\left[Z_{m+1} \mid U_{m+1}\right]\right)\right] \\
& =E\left[\phi\left(U_{m+1}\right)\right] ;
\end{aligned}
$$

and this proves that for $m \geq 2, \mu_{F_{m}}$ is strictly decreasing. In addition, recall that $\mu_{F_{1}}=1 / \alpha>\mu_{F_{2}}$; therefore, it also follows that $\mu_{F_{m}}$ is finite for all $m$.

To determine the limit of the $\mu_{F_{m}} \mathrm{~s}$, observe that (31) can be written as

$$
\mu_{F_{m}}=\frac{m-1}{m} s_{m-1}+\frac{1}{m} \frac{1}{\alpha+\beta},
$$

where

$$
s_{m-1} \equiv \frac{1}{m-1} \sum_{i=0}^{m-2} \frac{1}{\alpha+\beta i /(m-1)} .
$$


Now, the fact that the function $\phi$ is decreasing implies that $s_{m-1}$ is an upper Riemann sum of $\phi$ in the interval $[0,1]$. Since $\phi$ is integrable, it follows that $s_{m-1}$ converges to

$$
\int_{0}^{1} \phi(y) \mathrm{d} y=\int_{0}^{1} \frac{1}{\alpha+\beta y} \mathrm{~d} y=\frac{1}{\beta} \ln \left(\frac{\alpha+\beta}{\alpha}\right),
$$

which is $\mu_{F_{\infty}}$ (see (27)); and this, together with the fact that the second term in (35) converges to 0 , proves that

$$
\lim _{m \rightarrow \infty} \mu_{F_{m}}=\mu_{F_{\infty}}
$$

Finally, we return to (25) and rewrite its right-hand side as

$$
\int_{0}^{\infty}\left[\bar{F}_{m}(t)-\bar{G}_{m}(t)\right] \mathrm{d} t=\left(\mu_{F_{m}}-\mu_{F_{\infty}}\right)+\left(\mu_{F_{\infty}}-\mu_{G_{m}}\right) .
$$

It now follows from (36) and (28) that both $\mu_{F_{m}}-\mu_{F_{\infty}}$ and $\mu_{F_{\infty}}-\mu_{G_{m}}$ converge to 0 as $m \rightarrow \infty$. Since we have also shown that the convergence is, for both cases, monotone, this completes the proof.

We are now ready to prove (11).

Proof of (11) We will establish, for all $t \geq 0$, the following two inequalities:

$$
F_{\infty}(t) \geq \limsup _{m \rightarrow \infty} F_{m}(t)
$$

and

$$
F_{\infty}(t) \leq \liminf _{m \rightarrow \infty} F_{m}(t)
$$

The convergence in (11) then follows from (37) and (38), since

$$
F_{\infty}(t) \leq \liminf _{m \rightarrow \infty} F_{m}(t) \leq \limsup _{m \rightarrow \infty} F_{m}(t) \leq F_{\infty}(t)
$$

(see, e.g., Rudin [18], pp. 56-57).

Consider (37) first. Since $G_{m}(t) \geq F_{m}(t)$ (Lemma 2), we have

$$
\limsup _{m \rightarrow \infty} G_{m}(t) \geq \limsup _{m \rightarrow \infty} F_{m}(t), \quad t \geq 0 .
$$

In the proof of Lemma 3, we showed that for all $t \geq 0, \bar{G}_{m}(t)$ converges monotonically from below to $\bar{F}_{\infty}(t)$. The convergence of $\bar{G}_{m}$, and hence of $G_{m}$, implies that

$$
\limsup _{m \rightarrow \infty} G_{m}(t)=\lim _{m \rightarrow \infty} G_{m}(t)=F_{\infty}(t)
$$

and this, together with (39), proves (37). 
We now turn our attention to (38), which we prove by contradiction. Consider an arbitrary fixed $t$, say $t^{*}$; and suppose (38) does not hold at $t^{*}$. Then, there exists a positive $\varepsilon$ and a subsequence $\left\{n_{k}\right\}_{k \geq 1}$ of positive integers such that

$$
F_{n_{k}}\left(t^{*}\right) \leq F_{\infty}\left(t^{*}\right)-\varepsilon
$$

for all $k \geq 1$. Now, consider the function $G_{n_{k}}$ and recall from Lemma 2 that $G_{n_{k}}(t) \geq F_{n_{k}}(t)$ for all $t \geq 0$. Moreover, as a consequence of Lemma 3, we have

$$
\lim _{k \rightarrow \infty} \int_{0}^{\infty}\left[G_{n_{k}}(t)-F_{n_{k}}(t)\right] \mathrm{d} t=0
$$

We will show that (40) is in contradiction with (41).

Suppose (40) holds. For any given $k$, define a distribution function $H_{n_{k}}$ as follows:

$$
H_{n_{k}}(t) \equiv\left\{\begin{array}{cl}
G_{n_{k}}(t), & \text { for } 0 \leq t<G_{n_{k}}^{-1}\left(F_{n_{k}}\left(t^{*}\right)\right) \\
F_{n_{k}}\left(t^{*}\right), & \text { for } G_{n_{k}}^{-1}\left(F_{n_{k}}\left(t^{*}\right)\right) \leq t<t^{*} \\
G_{n_{k}}(t), & \text { for } t^{*} \leq t<\infty
\end{array}\right.
$$

where the superscript " -1 " in $G_{n_{k}}^{-1}$ denotes functional inverse. Since $G_{n_{k}}(t)$ and $H_{n_{k}}(t)$ agree at $t=G_{n_{k}}^{-1}\left(F_{n_{k}}\left(t^{*}\right)\right)$ and at $t=t^{*}$ and since the function $G_{n_{k}}$ is strictly increasing in $t$, we have $G_{n_{k}}(t) \geq F_{n_{k}}\left(t^{*}\right)$ for $G_{n_{k}}^{-1}\left(F_{n_{k}}\left(t^{*}\right)\right) \leq t<t^{*}$; and therefore, $G_{n_{k}}(t) \geq H_{n_{k}}(t)$ for all $t \geq 0$. Moreover, since the function $F_{n_{k}}$ is strictly increasing in $t$, so that $F_{n_{k}}(t)<F_{n_{k}}\left(t^{*}\right)$ for $G_{n_{k}}^{-1}\left(F_{n_{k}}\left(t^{*}\right)\right) \leq t<t^{*}$, and since $G_{n_{k}}(t) \geq F_{n_{k}}(t)$ for all $t$, we also have that $H_{n_{k}}(t) \geq F_{n_{k}}(t)$ for all $t \geq 0$. Thus, the function $H_{n_{k}}$ is, by construction, sandwiched between $G_{n_{k}}$ and $F_{n_{k}}$. It follows that

$$
\int_{0}^{\infty}\left[G_{n_{k}}(t)-F_{n_{k}}(t)\right] \mathrm{d} t \geq \int_{0}^{\infty}\left[G_{n_{k}}(t)-H_{n_{k}}(t)\right] \mathrm{d} t .
$$

Next, observe that

$$
G_{n_{k}}^{-1}\left(F_{n_{k}}\left(t^{*}\right)\right) \leq G_{n_{k}}^{-1}\left(F_{\infty}\left(t^{*}\right)-\varepsilon\right)<F_{\infty}^{-1}\left(F_{\infty}\left(t^{*}\right)-\varepsilon\right)<F_{\infty}^{-1}\left(F_{\infty}\left(t^{*}\right)\right)=t^{*}
$$

and that these inequalities, together with (42), (40), and $G_{n_{k}}(t)>F_{\infty}(t)$, imply that the righthand side of (43) can be further bounded as follows:

$$
\begin{aligned}
\int_{0}^{\infty}\left[G_{n_{k}}(t)-H_{n_{k}}(t)\right] \mathrm{d} t & =\int_{G_{n_{k}}^{-1}\left(F_{n_{k}}\left(t^{*}\right)\right)}^{t^{*}}\left[G_{n_{k}}(t)-F_{n_{k}}\left(t^{*}\right)\right] \mathrm{d} t \\
& \geq \int_{F_{\infty}^{-1}\left(F_{\infty}\left(t^{*}\right)-\varepsilon\right)}^{t^{*}}\left[G_{n_{k}}(t)-F_{n_{k}}\left(t^{*}\right)\right] \mathrm{d} t \\
& \geq \int_{F_{\infty}^{-1}\left(F_{\infty}\left(t^{*}\right)-\varepsilon\right)}^{t^{*}}\left[F_{\infty}(t)-\left(F_{\infty}\left(t^{*}\right)-\varepsilon\right)\right] \mathrm{d} t .
\end{aligned}
$$

Finally, since $F_{\infty}$ is strictly increasing and since $F_{\infty}^{-1}\left(F_{\infty}\left(t^{*}\right)-\varepsilon\right)<t^{*}$, the last bound in (44) is positive; moreover, this lower bound is independent of $k$. We have, therefore, arrived at a contradiction to (41). This establishes (38), and the proof is complete. 
Denote by $\delta_{m}(t)$ the variance of the total number of adoptions by time $t$; that is, let $\delta_{m}(t) \equiv \operatorname{Var}\left[A_{m}(t)\right]$. It is easily seen that $\delta_{1}(t)=F_{1}(t)\left[1-F_{1}(t)\right]$. For $m \geq 2$, we derive in the next lemma a formula for $\delta_{m}(t)$.

LEMMA 4 For $m \geq 2$, we have

$$
\delta_{m}(t)=\frac{m^{2}}{\beta}\left\{\left[1-F_{m}(t)\right]\left[\alpha+\beta F_{m}(t)\right]-f_{m}(t)\right\}+\frac{m}{\beta}\left\{f_{m}(t)-\alpha\left[1-F_{m}(t)\right]\right\}, \quad t \geq 0 .
$$

Proof Upon taking expectations, (15) becomes

$$
E\left[\lambda_{m A_{m}(t)}\right]=\alpha\left\{m-E\left[A_{m}(t)\right]\right\}+\frac{\beta}{m-1}\left\{m E\left[A_{m}(t)\right]-E\left\{\left[A_{m}(t)\right]^{2}\right\}\right\}
$$

which, after a rearrangement, yields

$$
E\left\{\left[A_{m}(t)\right]^{2}\right\}=\frac{(m-1) \alpha}{\beta}\left\{m-E\left[A_{m}(t)\right]\right\}+m E\left[A_{m}(t)\right]-\frac{m-1}{\beta} E\left[\lambda_{m A_{m}(t)}\right] .
$$

Hence,

$$
\begin{aligned}
\delta_{m}(t) & =E\left\{\left[A_{m}(t)^{2}\right\}-\left\{E\left[A_{m}(t)\right]\right\}^{2}\right. \\
& =\left\{\frac{(m-1) \alpha}{\beta}+E\left[A_{m}(t)\right]\right\}\left\{m-E\left[A_{m}(t)\right]\right\}-\frac{m-1}{\beta} E\left[\lambda_{\left.m A_{m}(t)\right]} .\right.
\end{aligned}
$$

Upon substitution of $E\left[A_{m}(t)\right]=m F_{m}(t)$ and $E\left[\lambda_{m A_{m}(t)}\right]=m f_{m}(t)$ (see (17)), the last expression rearranges straightforwardly to (45).

We are finally in position to prove (12), and hence to complete the proof of the Theorem.

Proof of (12) From (8) and (45), we have

$$
\begin{aligned}
\operatorname{Var}\left[B_{m}(t)\right] & =\frac{1}{m^{2}} \delta_{m}(t) \\
& =\frac{1}{\beta}\left\{\left[1-F_{m}(t)\right]\left[\alpha+\beta F_{m}(t)\right]-f_{m}(t)\right\}+\frac{1}{m \beta}\left\{f_{m}(t)-\alpha\left[1-F_{m}(t)\right]\right\} .
\end{aligned}
$$

Since $F_{\infty}(t)$ satisfies (3) with $p=\alpha$ and $q=\beta$, the convergence in (11) implies that

$$
\lim _{m \rightarrow \infty}\left\{\left[1-F_{m}(t)\right]\left[\alpha+\beta F_{m}(t)\right]-f_{m}(t)\right\}=0 .
$$

Next, it is easily seen from (13) that $f_{m}(t)-\alpha\left[1-F_{m}(t)\right]$ is uniformly bounded; and hence,

$$
\lim _{m \rightarrow \infty} \frac{1}{m \beta}\left\{f_{m}(t)-\alpha\left[1-F_{m}(t)\right]\right\}=0
$$

Finally, (46), (47), and (48) together yield (12). 


\section{COMMENTS}

Since the publication of Bass's paper, the solution (4), or its derivative,

$$
f(t)=\frac{\left[(p+q)^{2} / p\right] e^{-(p+q) t}}{\left[1+(q / p) e^{-(p+q) t}\right]^{2}},
$$

has been used extensively to forecast the growth of sales volume of new products over time. In such applications, it is important to develop estimates for the parameters $p$ and $q$ from (say) historical data (see, e.g., Putsis and Srinivasan [14], Section 11.2.1). A standard framework for this purpose is to conduct a regression analysis (ordinary least squares or nonlinear least squares) based on the assumption that the actual sales in successive time intervals can be modelled as the sum of two independent components: the (discretized) adoption-rate curve, $m f(t)$, and a sequence of i.i.d. error terms. The adoption of such a framework can be attributed to the fact that the BM assumes that $N(t)$ is a deterministic function. In other words, the deterministic assumption effectively forces one to model deviations of the actual sales data from the adoption-rate curve (solely) as manifestations of the presence of independent random errors, as opposed to being a consequence of the underlying stochastic nature of the forces behind successive adoptions. Further work to investigate the use of SBM as a framework for parameter estimation will be reported in a subsequent paper.

The formula for $\delta_{m}(t)$, the variance of cumulative adoptions, in Lemma 4 is potentially useful in applications where the variability of the cumulative-adoptions curve is of importance (see, e.g., Cohen, Ho, and Matsuo [6], p. 245). The asymptotic form of this variance function is also relevant in parameter estimation. A natural conjecture is that the growth of $\delta_{m}(t)$ is of order $m$ and $\sqrt{m}\left[B_{m}(t)-F_{\infty}(t)\right]$ is normally distributed as $m \rightarrow \infty$.

\section{Acknowledgements}

This research is supported in part by a Special Faculty Development Assignment program from The University of Texas at Dallas. The author is grateful to his colleagues F. M. Bass and R. Chandrasekaran for numerous stimulating discussions.

\section{References}

[1] Bailey, N. T. J. (1950) A simple stochastic epidemic, Biometrika, 37, 193-202.

[2] Bailey, N. T. J. (1975) The Mathematical Theory of Infectious Diseases and Its Applications (2nd ed.). London and High Wycombe: Griffin.

[3] Bartholomew, D. J. (1982) Stochastic Models for Social Processes (3rd ed.). John Wiley \& Sons.

[4] Bartlett, M. S. (1960) Stochastic Population Models in Ecology and Epidemiology. London: Methuen.

[5] Bass, F. M. (1969) A new product growth model for consumer durables, Management Science, 15, $215-227$

[6] Cohen, M. A., Ho, T. H. and Matsuo, H. (2000) Operations planning in the presence of innovation-diffusion dynamics, In: Mahajan, V., Muller, E. and Wind, E. (Eds.), New Product Diffusion Models. Boston: Kluwer Academic Publishers, pp. 237-259.

[7] Eliashberg, J. and Chatterjee, R. (1986) Stochastic issues in innovation diffusion models, In: Mahajan, V. and Wind, Y. (Eds.), Innovation Diffusion Models of New-Product Acceptance. Cambridge, MA: Ballinger, pp. 151-203.

[8] Feller, W. (1934) Die Grundlagen der Volterraschen Theorie des Kampfes ums Dasein in wahrscheinlichkeitstheoretischer Behandlung, Acta Biotheoretica, 5, 11-40.

[9] Gani, J. (1972) Point processes in epidemiology, In: Lewis, P. A. W. (Ed.), Stochastic Point Processes: Statistical Analysis, Theory, and Applications. New York: Wiley.

[10] Haskey, H. W. (1954) A general expression for the mean in a simple stochastic epidemic, Biometrika, 41, $272-275$. 
[11] Mahajan, V., Muller, E. and Wind, Y. (2000) New Product Diffusion Models. Boston: Kluwer Academic Publishers.

[12] Mahajan, V. and Wind, Y. (1986) Innovation Diffusion Models of New-Product Acceptance. Cambridge, MA: Ballinger.

[13] Mansfield, E. and Hensley, C. (1960) The logistic process: Tables of the stochastic epidemic curve and applications, Journal of The Royal Statistical Society, B22, 332-337.

[14] Putsis, W. P. Jr., and Srinivasan, V. (2000) Estimation techniques for macro diffusion models, In: Mahajan, V., Muller, E. and Wind, Y. (Eds.), New Product Diffusion Models. Boston: Kluwer Academic Publishers, pp. 263-291.

[15] Roberts, J. H. and Lattin, J. M. (2000) Disaggregate-level diffusion models, In: Mahajan, V., Muller, E. and Wind, Y. (Eds.), New Product Diffusion Models. Boston: Kluwer Academic Publishers, pp. 207-236.

[16] Rogers, E. M. (1995) Diffusion of Innovations, 4th ed. New York: Free Press.

[17] Ross, S. M. (2000) Introduction to Probability Models, 7th ed. Academic Press.

[18] Rudin, W. (1976) Principles of Mathematical Analysis, 3rd ed. McGraw-Hill.

[19] Taga, Y. and Isii, K. (1959) On a stochastic model concerning the pattern of communication - diffusion of news in a social group, Annals of The Institute of Statistical Mathematics, 11, 25-43.

[20] Williams, T. (1965) The simple stochastic epidemic curve for large populations of susceptibles, Biometrika, 52, $571-579$ 